\title{
Establishing a Plan of Action Implementing Integrated Disease Surveillance in Sudan
}

\author{
Hayat Khogali', Ngozi A. Erondu*2, 4, Betiel H. Haile ${ }^{4}$ and Scott J. McNabb ${ }^{3,4}$ \\ 'Sudan Federal Ministry of Health, Khartoum, Sudan; 'London School of Hygiene and Tropical Medicine, London, United Kingdom; \\ ${ }^{3}$ Emory University, Rollins School of Public Health, Atlanta, GA, USA; ${ }^{4}$ Public Health Practice, LLC, Atlanta, GA, USA
}

\section{Objective}

This presentation aims to discuss the need and share the results from an international, multi-disciplinary mission to assess public health surveillance (PHS) in Sudan and develop a plan of action (PoA) to strengthen PHS through integration. We will present the findings of the mission and the resulting PoA developed for Public Health Surveillance Strengthening (PHSS) designed to rapidly detect, report and respond to infectious diseases and establish sustainable, integrated One Health concepts.

\section{Introduction}

Integrated Disease Surveillance and Response (IDSR) is a strategy conceived and adopted by the World Health Organization Regional Office for Africa in 1998. The goal of IDSR is to support PHSS in Africa and enhance efficiency and communication across all levels of the national public health system. IDSR emphasizes the commitment of the revised International Health Regulations [IHR (2005)] to strengthen and maintain core capacities to detect, assess, report, and respond to public health events of international concern.

In February 2014, the Sudan Federal Ministry of Health (FMoH), requested an assessment of PHS with attention to PHSS integration opportunities and enhancing their ability to meet IHR (2005) requirements. A WHO Regional Office for the Eastern Mediterranean (WHO/EMRO) team of consultants performed this mission in collaboration with the FMoH.

\section{Methods}

The mission drew observations and conclusions and framed recommendations, derived from document reviews, semi-structured interviews with key informants, discussions with heads of diseasespecific programs and through observations on how PHS data were collected and reported from the peripheral levels to higher levels, as seen during the field visits. The findings of this technical mission and the recommendations were formulated into a country report and PoA.

\section{Results}

The mission found that PHS in Sudan consisted of multiple, siloed, vertical disease PHS systems, each using separate data collection and reporting paper-based forms. This resulted in duplication of data collection and inconsistencies in reported data from various sources. It also creates a burden on staff. A PoA to implement and sustain PHSS through integration in Sudan was developed and recommended to the Sudan FMoH. The PoA advocated a strategic approach of PHSS, which incorporated IHR (2005) and One Health concepts.

This PoA included technical, training, and management support services required to establish PHSS, e-Surveillance, and a Field Epidemiology Training Program (FETP) in Sudan. The main activities of this approach included:

- Facilitate coordination of national entities for One Health PHSS with an objective of early warning and response

- Develop and modernize PHS tools, standards, guidelines and policies, and streamline reporting channels
- Develop policies, and framework for national laboratory PHSS, including standards and guidelines

- Train health and PHS officers on PHSS components

- Develop an FETP introduction plan

\section{Conclusions}

Empowering PHSS will equip a motivated health staff with a strategy to streamline PHS functions; coordinate an "all-hazards" monitoring and alert approach; and provide national consistency for detection, analyses, and reporting. If implemented, the recommended PoA supports a long-term goal to shift - in a phased manner - from the present fragmented, disease-specific, PHS focus, to an integrated one with full implementation capacity at the community, local, state, and national levels.

\section{Keywords}

IDSR; IHR(2005); Sudan; Plan of Action; One Health

\section{Acknowledgments}

The authors would like to greatly acknowledge the original mission members Dr. Hoda Mansour [HM] (NAMRU-3, Cairo) and Dr. Margaret Lamunu [ML] (WHO/HQ) as well as the support received from the CSR team of the WHO Coordinating Office and the Sudan FMoH.

\section{References}

McNabb, S.J.N., S. Chungong, M. Ryan, T. Wuhib, P. Nsubuga, W. Alemu, V. Carande-Kulis, and G. Rodier. Conceptual Framework of Public Health Surveillance and Action and its Application in Health Sector Reform. BMC Public Health 2002,2:2

McNabb, S.J.N., S. Chungong, M. Ryan, T. Wuhib, P. Nsubuga, W. Alemu, V. Carande-Kulis, and G. Rodier. Conceptual Framework of Public Health Surveillance and Action and its Application in Health Sector Reform. BMC Public Health 2002,2:2

Technical Guidelines for Integrated Disease and Response in the African Region (2nd Edition) October 2012

\footnotetext{
*Ngozi A. Erondu

E-mail: ngozierondu@gmail.com
} 